\title{
Strong commutativity preserving derivations on Lie ideals of prime $\Gamma$-rings
}

\author{
Okan Arslan, Berna Arslan*
}

\begin{abstract}
Let $M$ be a $\Gamma$-ring and $S \subseteq M$. A mapping $f: M \rightarrow M$ is called strong commutativity preserving on $S$ if $[f(x), f(y)]_{\alpha}=[x, y]_{\alpha}$, for all $x, y \in S, \alpha \in \Gamma$. In the present paper, we investigate the commutativity of the prime $\Gamma$-ring $M$ of characteristic not 2 with center $Z(M) \neq(0)$ admitting a derivation which is strong commutativity preserving on a nonzero square closed Lie ideal $U$ of $M$. Moreover, we also obtain a related result when a mapping $d$ is assumed to be a derivation on $U$ satisfying the condition $d(u) \circ_{\alpha} d(v)=u \circ_{\alpha} v$, for all $u, v \in U$, $\alpha \in \Gamma$.
\end{abstract}

\section{INTRODUCTION}

Nobusawa [13] developed the concept of a gamma ring and then Barnes [1] weakened slightly the defining conditions for a gamma ring. After these definitions a number of mathematicians have studied on gamma rings in the sense of Barnes and Nobusawa and get results parallel to the ring theory (see for example [1], [11], [9]).

Let $R$ be any ring. The symbol $[a, b]$ denotes $a b-b a$ for $a, b \in R$. $R$ is called prime if $a R b=(0)$ implies either $a=0$ or $b=0$, and $R$ is called semiprime if $a R a=(0)$ implies $a=0$. An additive mapping $d$ is called a derivation on $R$ if

$$
d(a b)=d(a) b+a d(b)
$$

holds for all $a, b \in R$.

A mapping $f$ is said to be commutativity preserving on $R$ if $[f(a), f(b)]=0$ whenever $[a, b]=0$, for all $a, b \in R$. In 1976, Watkins [14] obtained the first result on commutativity preserving maps for a $n \times n$ matrix algebra when $n \geq 4$ and $f$ is a monomorphism on $R$. Recently, the study of commutativity preserving maps has become an active research area in ring theory (see for example [4], [6], [8], [12] and references therein).

2010 Mathematics Subject Classification. Primary: 16W25; Secondary: 16N60, 16 Y99.

Key words and phrases. Prime gamma rings, Lie ideals, derivations, strong commutativity preserving maps.

Full paper. Received 3 July 2018, accepted 7 April 2019, available online 25 April 2019.

*Corresponding Author 
Let $S$ be a subset of $R$. A map $f$ is called strong commutativity preserving (SCP) on $S$ if $[f(a), f(b)]=[a, b]$, for all $a, b \in S$. Clearly, a map that is strong commutativity preserving on a set $S$ is also commutativity preserving on $S$, but the inverse is not true in general. The notion of a strong commutativity preserving map was first introduced by H.E. Bell and G. Mason [3]. Later, H.E. Bell and M.N. Daif [2] proved that if a semiprime ring $R$ admits a nonzero derivation which is strong commutativity preserving on a right ideal $\rho$ of $R$, then $\rho \subseteq Z(R)$ where $Z(R)$ is the center of $R$. In particular, $R$ is commutative if $\rho=R$. M. Brešar and C.R. Miers [5] characterized SCP additive maps on a semiprime ring. In [10], Brešar and Miers's result was extended to Lie ideals of prime rings by J.-S. Lin and C.-K. Liu. Later, Q. Deng and M. Ashraf [7] proved that if there exists a derivation $d$ of a semiprime ring $R$ and a mapping $f: I \rightarrow R$ defined on a nonzero ideal $I$ of $R$ such that $[f(a), d(b)]=[a, b]$, for all $a, b \in I$, then $R$ contains a nonzero central ideal. They also showed that $R$ is commutative when $I=R$. There are lots of generalizations similar to these results can be found in the literature.

Recently, X. Xu, J. Ma and Y. Zhou [15] proved that a semiprime $\Gamma$ ring with a strong commutativity preserving derivation on itself must be commutative and that a strong commutativity preserving endomorphism $\sigma$ on a semiprime $\Gamma$-ring $M$ must have the form $\sigma(a)=a+\xi(a)(a \in M)$ where $\xi$ is a map from $M$ into its center, which extends some results by Bell and Daif to semiprime $\Gamma$-rings.

Motivated by all these results, in the present paper, we study strong commutativity preserving derivations on a nonzero square closed Lie ideal of prime $\Gamma$-rings and prove that if $M$ is a prime $\Gamma$-ring of characteristic not 2 such that its center $Z(M) \neq(0)$ and $d$ is a SCP derivation on a nonzero square closed Lie ideal $U$ of $M$, then $U \subseteq Z(M)$. In particular, $M$ is commutative if $U=M$. Moreover, we also obtain the same result when a mapping $d$ is assumed to be a derivation on $U$ satisfying the condition $d(u) \circ_{\alpha} d(v)=u \circ_{\alpha} v$, for all $u, v \in U, \alpha \in \Gamma$.

\section{Preliminaries}

Before giving our results, we first present some preliminary definitions. In this paper, $M$ will represent a $\Gamma$-ring in the sense of Barnes [1] unless otherwise stated.

An additive subgroup $K$ of a $\Gamma$-ring $M$ is called a left (resp. right) ideal of $M$ if $M \Gamma K \subseteq K$ (resp. $K \Gamma M \subseteq K$ ). A left ideal $K$ of a $\Gamma$-ring $M$ is called an ideal of $M$ if it is also a right ideal of $M$. The set of all elements $a$ satisfying $a \alpha b=b \alpha a$ for all $b \in M$ and $\alpha \in \Gamma$ is called the center of $M$.

A $\Gamma$-ring $M$ is said to be prime if $a \Gamma M \Gamma b=(0)$ for $a, b \in M$ implies that $a=0$ or $b=0$. An additive mapping $d$ is called a derivation on $M$ if $d(a \alpha b)=d(a) \alpha b+a \alpha d(b)$, for all $a, b \in M$ and $\alpha \in \Gamma$. 
Let $M$ be a $\Gamma$-ring and $a, b \in M, \alpha \in \Gamma$. The commutator of $a$ and $b$ with respect to $\alpha$ is defined as the element $a \alpha b-b \alpha a$ and denoted by $[a, b]_{\alpha}$. According to this definition we have the following equations,

$$
\begin{aligned}
& {[a \alpha b, c]_{\beta}=[a, c]_{\beta} \alpha b+a \alpha[b, c]_{\beta}+a \alpha c \beta b-a \beta c \alpha b,} \\
& {[a, b \alpha c]_{\beta}=[a, b]_{\beta} \alpha c+b \alpha[a, c]_{\beta}+b \beta a \alpha c-b \alpha a \beta c,}
\end{aligned}
$$

where $a, b, c \in M, \alpha, \beta \in \Gamma$. Similarly, the anti-commutator of $a$ and $b$ with respect to $\alpha$ is defined as the element $a \alpha b+b \alpha a$ and denoted by $a \circ_{\alpha} b$. According to this definition we have the following equations,

$$
\begin{aligned}
(a \alpha b) \circ_{\beta} c & =a \alpha\left(b \circ_{\beta} c\right)-[a, c]_{\beta} \alpha b+a \alpha c \beta b-a \beta c \alpha b \\
& =\left(a \circ_{\beta} c\right) \alpha b+a \alpha[b, c]_{\beta}+a \beta c \alpha b-a \alpha c \beta b, \\
a \circ_{\beta}(b \alpha c) & =\left(a \circ_{\beta} b\right) \alpha c-b \alpha[a, c]_{\beta}+b \beta a \alpha c-b \alpha a \beta c \\
& =b \alpha\left(a \circ_{\beta} c\right)+[a, b]_{\beta} \alpha c+b \alpha a \beta c-b \beta a \alpha c,
\end{aligned}
$$

where $a, b, c \in M, \alpha, \beta \in \Gamma$.

An additive subgroup $U$ of a $\Gamma$-ring $M$ is called a Lie ideal if $[u, m]_{\alpha} \in U$, for all $u \in U, m \in M$ and $\alpha \in \Gamma$. A Lie ideal $U$ of $M$ is said to be a square closed Lie ideal of $M$, if $u \alpha u \in U$ for all $u \in U$ and $\alpha \in \Gamma$. Clearly, $u \alpha v+v \alpha u \in U$, for all $u, v \in U, \alpha \in \Gamma$. Similarly, we have $u \alpha v-v \alpha u \in U$. Moreover, by using these relations, we get $2 u \alpha v \in U$ which will be used in the whole paper frequently.

A map $f$ from a $\Gamma$-ring $M$ into itself is called strong commutativity preserving (SCP) on a subset $S$ of $M$ if $[f(a), f(b)]_{\alpha}=[a, b]_{\alpha}$ holds for all $a, b \in S$ and $\alpha \in \Gamma$.

\section{The Results}

First, we work on SCP derivations on Lie ideals of prime $\Gamma$-rings. The following lemma will play an crucial role in the proofs of our main theorems.

Lemma 3.1. Let $M$ be a prime $\Gamma$-ring and $Z(M) \neq(0)$. Then the equations

$$
\begin{aligned}
& {[a \alpha b, c]_{\beta}=[a, c]_{\beta} \alpha b+a \alpha[b, c]_{\beta},} \\
& {[a, b \alpha c]_{\beta}=[a, b]_{\beta} \alpha c+b \alpha[a, c]_{\beta}}
\end{aligned}
$$

hold for all $a, b, c \in M, \alpha, \beta \in \Gamma$.

Proof. For any $c \in M, \alpha, \beta \in \Gamma$, the symbol $[\alpha, \beta]_{c}$ denotes $\alpha c \beta-\beta c \alpha$. Then, the commutator formulas in (1) and (2) become

$$
[a \alpha b, c]_{\beta}=[a, c]_{\beta} \alpha b+a \alpha[b, c]_{\beta}+a[\alpha, \beta]_{c} b
$$

and

$$
[a, b \alpha c]_{\beta}=[a, b]_{\beta} \alpha c+b \alpha[a, c]_{\beta}+b[\beta, \alpha]_{a} c,
$$

for all $a, b, c \in M, \alpha, \beta \in \Gamma$. 
Since $Z(M) \neq(0)$, there exists a nonzero element $x$ in $Z(M)$. Thus,

$$
\begin{aligned}
x \gamma y \delta a \alpha c \beta b & =y \gamma x \delta a \alpha c \beta b=y \gamma a \delta x \alpha c \beta b \\
& =y \gamma a \delta c \alpha x \beta b=y \gamma a \delta c \alpha b \beta x \\
& =y \gamma a \delta x \beta c \alpha b=y \gamma x \delta a \beta c \alpha b \\
& =x \gamma y \delta a \beta c \alpha b,
\end{aligned}
$$

for all $a, b, c, y \in M, \alpha, \beta, \gamma, \delta \in \Gamma$. Then we have that

$$
x \gamma y \delta a[\alpha, \beta]_{c} b=0,
$$

for all $a, b, c, y \in M, \alpha, \beta, \gamma, \delta \in \Gamma$. Multiplying the two sides of (3) by $x \gamma y \delta$ from the left hand side, and then comparing with (4) we get for all $a, b, c, y \in M, \alpha, \beta, \gamma, \delta \in \Gamma$

$$
x \gamma y \delta[a \alpha b, c]_{\beta}=x \gamma y \delta[a, c]_{\beta} \alpha b+x \gamma y \delta a \alpha[b, c]_{\beta} .
$$

That is $x \Gamma M \Gamma\left([a \alpha b, c]_{\beta}-[a, c]_{\beta} \alpha b-a \alpha[b, c]_{\beta}\right)=0$, for all $a, b, c \in M$, $\alpha, \beta \in \Gamma$. Since $M$ is prime and $x$ is nonzero, we have

$$
[a \alpha b, c]_{\beta}-[a, c]_{\beta} \alpha b-a \alpha[b, c]_{\beta}=0,
$$

for all $a, b, c \in M, \alpha, \beta \in \Gamma$. For the second equation, one can use the same method above, and this completes the proof.

Now, we can give a similar result for the anti-commutator formulas of $\Gamma$-rings.

Lemma 3.2. Let $M$ be a prime $\Gamma$-ring in the sense of Barnes and $Z(M) \neq(0)$. Then the equations

$$
\begin{aligned}
(a \alpha b) \circ_{\beta} c & =a \alpha\left(b \circ_{\beta} c\right)-[a, c]_{\beta} \alpha b \\
& =\left(a \circ_{\beta} c\right) \alpha b+a \alpha[b, c]_{\beta}, \\
a \circ_{\beta}(b \alpha c) & =\left(a \circ_{\beta} b\right) \alpha c-b \alpha[a, c]_{\beta} \\
& =b \alpha\left(a \circ_{\beta} c\right)+[a, b]_{\beta} \alpha c
\end{aligned}
$$

hold for all $a, b, c \in M, \alpha, \beta \in \Gamma$.

Proof. It can be proved by using the techniques of Lemma 3.1.

We need the following results to prove our main theorems.

Lemma 3.3. Let $M$ be a prime $\Gamma$-ring of characteristic not 2 with the center $Z(M) \neq(0)$ and $U$ be a Lie ideal of $M$. If $U \nsubseteq Z(M)$, then there exists an ideal $K$ of $M$ such that $[K, M]_{\Gamma} \subseteq U$ but $[K, M]_{\Gamma} \nsubseteq Z(M)$.

Proof. First, we show that the Lie product of $\mathrm{U}$ by itself is different from zero. Suppose that $[U, U]_{\Gamma}=(0)$. Then we have $\left[a,[a, m]_{\alpha}\right]_{\beta}=0$, for all $a \in U, m \in M$ and $\alpha, \beta \in \Gamma$. Replacing $m$ by $m \gamma x$ for $\gamma \in \Gamma$ and $x \in M$, we get

$$
[a, m]_{\beta} \gamma[a, x]_{\alpha}+[a, m]_{\alpha} \gamma[a, x]_{\beta}=0 .
$$


Now, replacing $\beta$ by $\alpha$ in (5) we have $[a, m]_{\alpha} \gamma[a, x]_{\alpha}=0$, for all $a \in U$, $m, x \in M$ and $\alpha, \gamma \in \Gamma$. Replacing $x$ by $y \delta x$ for $y \in M$ and $\delta \in \Gamma$ in the last equation, we get $[a, m]_{\alpha} \Gamma M \Gamma[a, x]_{\alpha}=(0)$, for all $a \in U, m, x \in M$ and $\alpha \in \Gamma$. Therefore, we have $U \subseteq Z(M)$ since $M$ is prime. But this contradicts with the hypothesis of the theorem. Hence, there exist $u, v \in U$ and $\beta \in \Gamma$ such that $[u, v]_{\beta} \neq 0$.

Let $K:=M \Gamma[u, v]_{\beta} \Gamma M$ and $T(U):=\left\{x \in M \mid[x, M]_{\Gamma} \subseteq U\right\}$. Then, it is clear that $K \neq(0)$ is an ideal of $M ; T(U)$ is a Lie ideal and a subring of $M$. Moreover, $U \subseteq T(U)$. Since $[u, v \gamma m]_{\beta}=[u, v]_{\beta} \gamma m+v \gamma[u, m]_{\beta}$ for all $m \in M$ and $\gamma \in \Gamma$, we get $[u, v]_{\beta} \Gamma M \subseteq T(U)$. Hence,

$$
\left[[u, v]_{\beta} \alpha m, n\right]_{\gamma} \in T(U),
$$

for all $n, m \in M$ and $\alpha, \gamma \in \Gamma$. Expanding this we get $n \gamma[u, v]_{\beta} \alpha m \in T(U)$ for all $n, m \in M$ and $\alpha, \gamma \in \Gamma$. Then, we have $M \Gamma[u, v]_{\beta} \Gamma M=K \subseteq T(U)$ which yields to $[K, M]_{\Gamma} \subseteq U$.

Now, suppose $[K, M]_{\Gamma} \subseteq Z(M)$. Therefore, we have $\left[K,[K, M]_{\Gamma}\right]_{\Gamma}=(0)$ and using the same argument above we get $K \subseteq Z(M)$. Let $x \in M$. Then $n \alpha k \gamma m \in K$ for all $n, m \in M, k \in K$ and $\alpha, \gamma \in \Gamma$. Since $K \subseteq Z(M)$ we have $[x, n \alpha k \gamma m]_{\delta}=0$. Expanding this we get $K \Gamma M \Gamma[x, M]_{\Gamma}=(0)$. Therefore, $x \in Z(M)$ since $M$ is prime and $K \neq(0)$. But this contradicts with $U \nsubseteq Z(M)$. This completes the proof.

Lemma 3.4. Let $M$ be a prime $\Gamma$-ring of characteristic not 2 with the center $Z(M) \neq(0)$ and $U$ be a Lie ideal of $M$. If $U \nsubseteq Z(M)$ and $a, b \in M$ such that $a \Gamma U \Gamma b=(0)$, then either $a=0$ or $b=0$.

Proof. By Lemma 3.3, there exists an ideal $K$ of $M$ such that $[K, M]_{\Gamma} \subseteq U$ but $[K, M]_{\Gamma} \nsubseteq Z(M)$. Let $u \in U, k \in K, m \in M$ and $\alpha, \beta, \gamma \in \Gamma$. Then, we have

$$
[k \alpha a \beta u, m]_{\gamma} \in[K, M]_{\Gamma} \subseteq U
$$

It follows from that

$$
\begin{aligned}
0 & =a \lambda[k \alpha a \beta u, m]_{\gamma} \epsilon b=a \lambda k \alpha a \beta[u, m]_{\gamma} \epsilon b+a \lambda[k \alpha a, m]_{\gamma} \beta u \epsilon b \\
& =a \lambda k \alpha a \gamma m \beta u \epsilon b-a \lambda m \gamma k \alpha a \beta u \epsilon b \\
& =a \lambda k \alpha a \gamma m \beta u \epsilon b,
\end{aligned}
$$

for all $u \in U, k \in K, m \in M$ and $\alpha, \beta, \gamma, \lambda, \epsilon \in \Gamma$. Therefore, we get $a \Gamma K \Gamma a=(0)$ or $U \Gamma b=(0)$ since $M$ is prime. In the first case, we see that $a$ must be zero by using the primeness of $M$. In the second case, we get

$$
[u, m]_{\alpha} \gamma b=0,
$$

for all $u \in U, m \in M$ and $\alpha, \gamma \in \Gamma$. Expanding this we have

$$
[u \gamma b, m]_{\alpha}-u \gamma[b, m]_{\alpha}=0,
$$


that is $u \gamma m \alpha b=0$, for all $u \in U, m \in M$ and $\alpha, \gamma \in \Gamma$. Therefore, $b=0$ since $M$ is prime and $U \neq(0)$.

Lemma 3.5. Let $M$ be a prime $\Gamma$-ring with the center $Z(M) \neq(0)$ and $x \in M$. If $a \in Z(M)$ and $a \gamma x \in Z(M)$ for all $\gamma \in \Gamma$, then $a=0$ or $x \in Z(M)$.

Proof. Suppose that $a \neq 0$. Since $a \gamma x \in Z(M)$, we have $[a \gamma x, m]_{\delta}=0$ for all $m \in M$ and $\delta, \gamma \in \Gamma$. Expanding this we get $a \gamma[x, m]_{\delta}=0$. Replacing $m$ by $m \beta n$ for $n \in M$ and $\beta \in \Gamma$ we conclude that $x \in Z(M)$ since $M$ is prime. This completes the proof.

Lemma 3.6. Let $M$ be a prime $\Gamma$-ring of characteristic not 2 with the center $Z(M) \neq(0)$ and $U$ be a Lie ideal of $M$. If $[U, U]_{\Gamma} \subseteq Z(M)$, then $U \subseteq Z(M)$.

Proof. By hypothesis we have $\left[u,[u, x]_{\alpha}\right]_{\beta} \in Z(M)$ for all $u \in U, x \in M$ and $\alpha, \beta \in \Gamma$. Since

$$
\left[u,[u, x]_{\alpha}\right]_{\beta} \gamma u=\left[u,[u, x]_{\alpha} \gamma u\right]_{\beta}=\left[u,[u, x \gamma u]_{\alpha}\right]_{\beta}
$$

and $\left[u,[u, x \gamma u]_{\alpha}\right]_{\beta} \in[U, U]_{\Gamma}$, we have $\left[u,[u, x]_{\alpha}\right]_{\beta} \gamma u \in Z(M)$. Therefore, we get $\left[u,[u, x]_{\alpha}\right]_{\beta}=0$ or $u \in Z(M)$ by Lemma 3.5. Now, let $\left[u,[u, x]_{\alpha}\right]_{\beta}=0$ for all $x \in M, \alpha, \beta \in \Gamma$ and for some $u \in U$. Replacing $x$ by $x \gamma m$ we get

$$
[u, x]_{\beta} \gamma[u, m]_{\alpha}+[u, x]_{\alpha} \gamma[u, m]_{\beta}=0,
$$

for all $x, m \in M$ and $\alpha, \beta, \gamma \in \Gamma$. Replacing $\beta$ by $\alpha$ in the equation (6) we get $[u, x]_{\alpha} \gamma[u, m]_{\alpha}=0$ since $M$ is a $\Gamma$-ring of characteristic not 2. Replacing $m$ by $m \delta n$ for $n \in M, \delta \in \Gamma$ in the last equation, we conclude that $u \in Z(M)$ since $M$ is prime. Consequently, we see that $U$ must be a subset of $Z(M)$.

Theorem 3.1. Let $M$ be a prime $\Gamma$-ring of characteristic not 2 and $U$ be a nonzero square closed Lie ideal of $M$. If $d$ is a $S C P$ derivation on $U$, then $U \subseteq Z(M)$ or $Z(M)=(0)$.

Proof. Suppose that $Z(M) \neq(0)$. We have $[d(x), d(y)]_{\alpha}=[x, y]_{\alpha}$ for all $x, y \in U$ and $\alpha \in \Gamma$ by hypothesis. Replacing $y$ by $2 y \beta z$ for $z \in U$ and $\beta \in \Gamma$, we get

$$
[d(x), d(2 y \beta z)]_{\alpha}=[x, 2 y \beta z]_{\alpha},
$$

for all $x, y, z \in U$ and $\alpha, \beta \in \Gamma$. By applying Lemma 3.1, we expand the last equation and we get

$$
d(y) \beta[d(x), z]_{\alpha}+[d(x), y]_{\alpha} \beta d(z)=0,
$$

since $M$ is a $\Gamma$-ring of characteristic not 2. Replacing $z$ by $2 z \gamma t$ for $z, t \in U$ and $\gamma \in \Gamma$ in the equation (7) we obtain that

$$
\begin{array}{r}
d(y) \beta[d(x), z]_{\alpha} \gamma t+d(y) \beta z \gamma[d(x), t]_{\alpha}+[d(x), y]_{\alpha} \beta d(z) \gamma t \\
+[d(x), y]_{\alpha} \beta z \gamma d(t)=0,
\end{array}
$$


since $M$ is a $\Gamma$-ring of characteristic not 2. Multiplying the two sides of (7) by $\gamma t$ from the right hand side, we have

$$
d(y) \beta[d(x), z]_{\alpha} \gamma t+[d(x), y]_{\alpha} \beta d(z) \gamma t=0,
$$

for all $x, y, z, t \in U$ and $\alpha, \beta, \gamma \in \Gamma$. Comparing (9) with (8), we have that

$$
d(y) \beta z \gamma[d(x), t]_{\alpha}+[d(x), y]_{\alpha} \beta z \gamma d(t)=0,
$$

for all $x, y, z, t \in U$ and $\alpha, \beta, \gamma \in \Gamma$. Since $U$ is a nonzero square closed Lie ideal of $M$, we have $[U, U]_{\Gamma}$ is a nonzero square closed Lie ideal of $M$, too. Writing $t=d(x)$ for $x \in[U, U]_{\Gamma}$, we obtain that

$$
[d(x), y]_{\alpha} \beta z \gamma d^{2}(x)=0,
$$

for all $y, z \in U, x \in[U, U]_{\Gamma}$ and $\alpha, \beta, \gamma \in \Gamma$. If we replace $y$ by $d(y)$ for $y \in[U, U]_{\Gamma}$ in the equation (10), we obtain $[x, y]_{\alpha} \Gamma U \Gamma d^{2}(x)=(0)$ for all $x, y \in[U, U]_{\Gamma}$, and $\alpha \in \Gamma$ since $d$ is SCP on $U$. Therefore,

$$
[x, y]_{\alpha} \beta 2[m, z]_{\alpha} \Gamma U \Gamma[x, y]_{\alpha} \beta 2[m, z]_{\alpha}=(0),
$$

since

$$
[x, y]_{\alpha} \Gamma U \Gamma\left[d^{2}(x), d^{2}(y)\right]_{\alpha} \beta 2[m, z]_{\alpha}=(0),
$$

for all $x, y \in[U, U]_{\Gamma}, m \in M, z \in U$ and $\alpha, \beta \in \Gamma$. Since $M$ is a $\Gamma$-ring of characteristic not 2 , we have $[x, y]_{\alpha} \beta[m, z]_{\alpha}=0$ by Lemma 3.4. Replacing $m$ by $m \gamma t$ for $t \in M$ and $\gamma \in \Gamma$ we get

$$
[x, y]_{\alpha} \beta m \gamma[t, z]_{\alpha}=0,
$$

for all $x, y \in[U, U]_{\Gamma}, m, t \in M, z \in U$ and $\alpha, \beta, \gamma \in \Gamma$. By the primeness of the $\Gamma$-ring $M$, we get either $[x, y]_{\alpha}=0$ or $[t, z]_{\alpha}=0$, for all $x, y \in[U, U]_{\Gamma}$, $z \in U, t \in M$ and $\alpha \in \Gamma$. In the second case, we see that $z \in Z(M)$ that is $U \subseteq Z(M)$. In the first case, using Lemma 3.6, we have $[U, U]_{\Gamma} \subseteq Z(M)$. Consequently, applying Lemma 3.6 again, we get that $U \subseteq Z(M)$ which completes the proof.

In particular, if we take $U=M$, then Theorem 3.1 gives a commutativity criterion as follows.

Corollary 3.1. Let $M$ be a prime $\Gamma$-ring of characteristic not 2 and $d$ be a derivation of $M$. If $Z(M) \neq(0)$ and $d$ is $S C P$ on $M$, then $M$ is commutative.

Since we can use the similar techniques of Theorem 3.1, we can obtain the following theorems which partially generalize the result of Bell and Daif to prime $\Gamma$-rings.

Theorem 3.2. Let $M$ be a prime $\Gamma$-ring of characteristic not 2 and $U$ be a nonzero square closed Lie ideal of $M$. If $[d(x), d(y)]_{\alpha}=-[x, y]_{\alpha}$ for all $x, y \in U$ and $\alpha \in \Gamma$, then $U \subseteq Z(M)$ or $Z(M)=(0)$.

Proof. It can be proved easily by using the same method in Theorem 3.1. 
Corollary 3.2. Let $M$ be a prime $\Gamma$-ring of characteristic not 2 and $d$ be a derivation of $M$. If $Z(M) \neq(0)$ and $[d(x), d(y)]_{\alpha}=-[x, y]_{\alpha}$ for all $x, y \in M, \alpha \in \Gamma$, then $M$ is commutative.

Theorem 3.3. Let $M$ be a prime $\Gamma$-ring of characteristic not 2 and $U$ be a nonzero square closed Lie ideal of $M$. If $d$ is a derivation of $M$ such that $d(x) \circ_{\alpha} d(y)=x \circ_{\alpha} y$ for all $x, y \in U$ and $\alpha \in \Gamma$, then $U \subseteq Z(M)$ or $Z(M)=(0)$.

Proof. Suppose that $Z(M) \neq(0)$. By the hypothesis we obtain that

$$
d(x) \circ_{\alpha} d(y)-x \circ_{\alpha} y=0,
$$

for all $x, y \in U$ and $\alpha \in \Gamma$. Replacing $x$ by $2 x \beta z$ for $z \in U, \beta \in \Gamma$ in the equation (11) we get

$$
d(x) \beta[z, d(y)]_{\alpha}-[x, d(y)]_{\alpha} \beta d(z)+2 x \beta y \alpha z=0,
$$

since $M$ is a $\Gamma$-ring of characteristic not 2. Taking $2 z \gamma x$ for $z$ in the equation (12) we have

$$
\begin{array}{r}
d(x) \beta[z, d(y)]_{\alpha} \gamma x+d(x) \beta z \gamma[x, d(y)]_{\alpha}-[x, d(y)]_{\alpha} \beta d(z) \gamma x \\
-[x, d(y)]_{\alpha} \beta z \gamma d(x)+2 x \beta y \alpha z \gamma x=0,
\end{array}
$$

for all $x, y, z \in U, \alpha, \beta, \gamma \in \Gamma$. Multiplying the two sides of (12) by $\gamma x$ from the right hand side, we get

$$
d(x) \beta[z, d(y)]_{\alpha} \gamma x-[x, d(y)]_{\alpha} \beta d(z) \gamma x+2 x \beta y \alpha z \gamma x=0,
$$

for all $x, y, z \in U$ and $\alpha, \beta, \gamma \in \Gamma$. If we compare (13) and (14), we have that

$$
d(x) \beta z \gamma[x, d(y)]_{\alpha}-[x, d(y)]_{\alpha} \beta z \gamma d(x)=0,
$$

for all $x, y, z \in U$ and $\alpha, \beta, \gamma \in \Gamma$. Replacing $z$ by $2 z \sigma[x, d(y)]_{\alpha}$ for $y \in[U, U]_{\Gamma}$ and $\sigma \in \Gamma$ in the equation (15) we get

$$
d(x) \beta z \sigma[x, d(y)]_{\alpha} \gamma[x, d(y)]_{\alpha}-[x, d(y)]_{\alpha} \beta z \sigma[x, d(y)]_{\alpha} \gamma d(x)=0,
$$

since $M$ is a $\Gamma$-ring of characteristic not 2. Taking $\sigma$ for $\gamma$ in (15) we have

$$
d(x) \beta z \sigma[x, d(y)]_{\alpha}=[x, d(y)]_{\alpha} \beta z \sigma d(x) .
$$

If we use the equation (17) in the equation (16) we get

$$
[x, d(y)]_{\alpha} \beta z \sigma d(x) \gamma[x, d(y)]_{\alpha}=[x, d(y)]_{\alpha} \beta z \sigma[x, d(y)]_{\alpha} \gamma d(x)
$$

and so

$$
[x, d(y)]_{\alpha} \beta z \sigma\left[d(x),[x, d(y)]_{\alpha}\right]_{\gamma}=0,
$$

for all $x, z \in U, y \in[U, U]_{\Gamma}$ and $\alpha, \beta, \gamma, \sigma \in \Gamma$. Taking $\beta=\gamma$ in (18), we get

$$
[x, d(y)]_{\alpha} \gamma z \sigma\left[d(x),[x, d(y)]_{\alpha}\right]_{\gamma}=0,
$$


for all $x, z \in U, y \in[U, U]_{\Gamma}$ and $\alpha, \gamma, \sigma \in \Gamma$. Multiplying the equation (19) on the left by $d(x) \gamma$ for $x \in[U, U]_{\Gamma}$, we have

$$
d(x) \gamma[x, d(y)]_{\alpha} \gamma z \sigma\left[d(x),[x, d(y)]_{\alpha}\right]_{\gamma}=0 .
$$

Taking $2 d(x) \gamma z$ for $z$ in (19) we obtain that

$$
[x, d(y)]_{\alpha} \gamma d(x) \gamma z \sigma\left[d(x),[x, d(y)]_{\alpha}\right]_{\gamma}=0,
$$

for all $z \in U, x, y \in[U, U]_{\Gamma}$ and $\alpha, \gamma, \sigma \in \Gamma$ since $M$ is a $\Gamma$-ring of characteristic not 2. Subtracting (21) from (20) we see that

$$
\left[d(x),[x, d(y)]_{\alpha}\right]_{\gamma} \gamma z \sigma\left[d(x),[x, d(y)]_{\alpha}\right]_{\gamma}=0,
$$

for all $z \in U, x, y \in[U, U]_{\Gamma}$ and $\alpha, \gamma, \sigma \in \Gamma$. Therefore, by Lemma 3.4 we have that

$$
\left[d(x),[x, d(y)]_{\alpha}\right]_{\gamma}=0,
$$

for all $x, y \in[U, U]_{\Gamma}$ and $\alpha, \gamma \in \Gamma$. Replacing $z$ by $x$ for $x \in[U, U]_{\Gamma}$ and $\beta=\gamma$ in (12) and using the equation (22) we conclude that $x \Gamma[U, U]_{\Gamma} \Gamma x=(0)$ for all $x \in[U, U]_{\Gamma}$ since $M$ is a $\Gamma$-ring of characteristic not 2 . We know that $[U, U]_{\Gamma}$ is a nonzero square closed Lie ideal of $M$. So by using Lemma 3.4 we get either $x=0$ for all $x \in[U, U]_{\Gamma}$ or $[U, U]_{\Gamma} \subseteq Z(M)$. The first case contradicts with the hypothesis $[U, U]_{\Gamma} \neq(0)$. Then we have that $[U, U]_{\Gamma} \subseteq Z(M)$. Hence, applying Lemma 3.6 we obtain that $U \subseteq Z(M)$. This completes the proof.

Corollary 3.3. Let $d$ be a derivation of a prime $\Gamma$-ring $M$ of characteristic not 2. If $d(x) \circ_{\alpha} d(y)=x \circ_{\alpha} y$ for all $x, y \in M, \alpha \in \Gamma$ and $Z(M) \neq(0)$, then $M$ is commutative.

Theorem 3.4. Let $M$ be a prime $\Gamma$-ring of characteristic not 2 and $U$ be a nonzero square closed Lie ideal of $M$. If $d$ is a derivation of $M$ such that $d(x) \circ_{\alpha} d(y)=-\left(x \circ_{\alpha} y\right)$ for all $x, y \in U, \alpha \in \Gamma$, then $U \subseteq Z(M)$ or $Z(M)=(0)$.

Proof. Suppose that $Z(M) \neq(0)$. By the hypothesis we have that

$$
d(x) \circ_{\alpha} d(y)+x \circ_{\alpha} y=0,
$$

for all $x, y \in U$ and $\alpha \in \Gamma$. Replacing $x$ by $2 x \beta z$ for $z \in U, \beta \in \Gamma$ in the equation (23) we get

$$
d(x) \beta[z, d(y)]_{\alpha}-[x, d(y)]_{\alpha} \beta d(z)+2 x \beta z \alpha y=0,
$$

since $M$ is a $\Gamma$-ring of characteristic not 2 . Taking $2 z \gamma x$ for $x$ in the equation (24) we have

$$
\begin{array}{r}
d(z) \gamma x \beta[z, d(y)]_{\alpha}+z \gamma d(x) \beta[z, d(y)]_{\alpha}-[z, d(y)]_{\alpha} \gamma x \beta d(z) \\
-z \gamma[x, d(y)]_{\alpha} \beta d(z)+2 z \gamma x \beta z \alpha y=0 .
\end{array}
$$

Multiplying the two sides of (24) by $z \gamma$ from the left hand side, we get

$$
z \gamma d(x) \beta[z, d(y)]_{\alpha}-z \gamma[x, d(y)]_{\alpha} \beta d(z)+2 z \gamma x \beta z \alpha y=0,
$$


for all $x, y, z \in U$ and $\alpha, \beta, \gamma \in \Gamma$. If we compare (25) and (26), we have that

$$
d(z) \gamma x \beta[z, d(y)]_{\alpha}-[z, d(y)]_{\alpha} \gamma x \beta d(z)=0,
$$

for all $x, y, z \in U$ and $\alpha, \beta, \gamma \in \Gamma$. Replacing $x$ by $2 x \sigma[z, d(y)]_{\alpha}$ for $y \in[U, U]_{\Gamma}$ and $\sigma \in \Gamma$ in the equation (27) we get

$$
d(z) \gamma x \sigma[z, d(y)]_{\alpha} \beta[z, d(y)]_{\alpha}-[z, d(y)]_{\alpha} \gamma x \sigma[z, d(y)]_{\alpha} \beta d(z)=0,
$$

since $M$ is a $\Gamma$-ring of characteristic not 2. Taking $\sigma$ for $\beta$ in (27) we have

$$
d(z) \gamma x \sigma[z, d(y)]_{\alpha}=[z, d(y)]_{\alpha} \gamma x \sigma d(z) .
$$

If we use the equation (29) in the equation (28) we get

$$
[z, d(y)]_{\alpha} \gamma x \sigma d(z) \beta[z, d(y)]_{\alpha}=[z, d(y)]_{\alpha} \gamma x \sigma[z, d(y)]_{\alpha} \beta d(z)
$$

and so

$$
[z, d(y)]_{\alpha} \gamma x \sigma\left[d(z),[z, d(y)]_{\alpha}\right]_{\beta}=0,
$$

for all $x, z \in U, y \in[U, U]_{\Gamma}$ and $\alpha, \beta, \gamma, \sigma \in \Gamma$. Taking $\beta=\gamma$ in (30), we get

$$
[z, d(y)]_{\alpha} \gamma x \sigma\left[d(z),[z, d(y)]_{\alpha}\right]_{\gamma}=0,
$$

for all $x, z \in U, y \in[U, U]_{\Gamma}$ and $\alpha, \gamma, \sigma \in \Gamma$. Multiplying the equation (31) on the left by $d(z) \gamma$ for $z \in[U, U]_{\Gamma}$, we have

$$
d(z) \gamma[z, d(y)]_{\alpha} \gamma x \sigma\left[d(z),[z, d(y)]_{\alpha}\right]_{\gamma}=0 .
$$

Taking $2 d(z) \gamma x$ for $x$ in (31) we obtain that

$$
[z, d(y)]_{\alpha} \gamma d(z) \gamma x \sigma\left[d(z),[z, d(y)]_{\alpha}\right]_{\gamma}=0,
$$

for all $x \in U, y, z \in[U, U]_{\Gamma}$ and $\alpha, \gamma, \sigma \in \Gamma$ since $M$ is a $\Gamma$-ring of characteristic not 2. Subtracting (33) from (32) we see that

$$
\left[d(z),[z, d(y)]_{\alpha}\right]_{\gamma} \gamma x \sigma\left[d(z),[z, d(y)]_{\alpha}\right]_{\gamma}=0,
$$

for all $x \in U, y, z \in[U, U]_{\Gamma}$ and $\alpha, \gamma, \sigma \in \Gamma$. Therefore, by Lemma 3.4 we have that

$$
\left[d(z),[z, d(y)]_{\alpha}\right]_{\gamma}=0,
$$

for all $y, z \in[U, U]_{\Gamma}$ and $\alpha, \gamma \in \Gamma$. Then, the proof is completed by using the similar steps in the equation (22) in Theorem 3.3.

Corollary 3.4. Let $d$ be a derivation of a prime $\Gamma$-ring $M$ of characteristic not 2. If $d(x) \circ_{\alpha} d(y)=-\left(x \circ_{\alpha} y\right)$ for all $x, y \in M, \alpha \in \Gamma$ and $Z(M) \neq(0)$, then $M$ is commutative.

Acknowledgement. This research was supported by Adnan Menderes University Research Fund. Project Number: FEF-18003. 


\section{REFERENCES}

[1] W. E. Barnes, On the Г-rings of Nobusawa, Pacific J. Math., 18 (1966), 411-422.

[2] H. E. Bell, M. N. Daif, On commutativity and strong commutativity preserving maps, Can. Math. Bull., 37 (1994), 443-447.

[3] H. E. Bell, G. Mason, On derivations in near rings and rings, Math. J. Okayama Univ., 34 (1992), 135-144.

[4] M. Brešar, Commuting traces of biadditive mappings, commutativity-preserving mappings and Lie mappings, Trans. Am. Math. Soc., 335 (1993), 525-546.

[5] M. Brešar, C. R. Miers, Strong commutativity preserving maps of semiprime rings, Canad. Math. Bull., 37 (1994), 457-460.

[6] M. Brešar, P. Šemrl, Commutativity preserving linear maps on central simple algebras, J. Algebra, 284 (2005), 102-110.

[7] Q. Deng, M. Ashraf, On strong commutativity preserving maps, Results Math., 30 (1996), 259-263.

[8] G. Dolinar, P. Šemrl, Maps on matrix algebras preserving commutativity, Linear Multilinear Algebra, 52 (2004), 69-78.

[9] S. Kyuno, On prime Г-rings, Pacific J. Math., 75 (1978), 185-190.

[10] J.-S. Lin, C.-K. Liu, Strong commutativity preserving maps on Lie ideals, Linear Algebra Appl., 428 (2008), 1601-1609.

[11] J. Luh, On the theory of simple Г-rings, Michigan Math. J., 16 (1969), 65-75.

[12] L. Molnar, P. Šemrl, Non-linear commutativity preserving maps on self-adjoint operators, Q. J. Math., 56 (2005), 589-595.

[13] N. Nobusawa, On a generalization of the ring theory, Osaka J. Math., 1 (1964), 81-89.

[14] W. Watkins, Linear maps that preserve commuting pairs of matrices, Linear Algebra Appl., 14 (1976), 29-35.

[15] X. Xu, J. Ma, Y. Zhou, Left derivations and strong commutativity preserving maps on semiprime $\Gamma$-rings, Southeast Asian Bull. Math., 39 (5) (2015), 735-745.

\section{Okan Arslan}

Department of Mathematics

Adnan Menderes University

09010 AYDin

TURKEY

E-mail address: oarslan@adu.edu.tr

\section{Berna Arslan}

Department of Mathematics

Adnan Menderes University

09010 AYDIN

TURKEY

E-mail address: byorganci@adu.edu.tr 